\title{
The interaction between orientation and motion signals in moving oriented Glass patterns
}

\author{
Andrea Pavan*, Lucy M. Bimson, Martin G. Gall, Filippo Ghin and George Mather \\ University of Lincoln, School of Psychology, Brayford Pool Lincoln, LN6 7TS, UK
}

\section{*Corresponding Author}

Andrea Pavan

University of Lincoln

Brayford Pool

Lincoln

Lincolnshire

LN6 7TS

United Kingdom

Email: apavan@lincoln.ac.uk

Tel: +44(0)1522 886154

Short title: Motion-form integration in moving Glass patterns

Number of pages: 21

Number of figures: 5 


\title{
The interaction between orientation and motion signals in moving oriented Glass patterns
}

\begin{abstract}
Previous psychophysical evidence suggests that motion and orientation processing systems interact asymmetrically in the human visual system, with orientation information having a stronger influence on the perceived motion direction than vice-versa. To investigate the mechanisms underlying this motion-form interaction we used moving and oriented Glass patterns (GPs), which consist of randomly distributed dot pairs (dipoles) that induce the percept of an oriented texture. In Experiment 1 we varied the angle between dipole orientation and motion direction (conflict angle). In separate sessions participants either judged the orientation or motion direction of the GP. In addition, the spatiotemporal characteristics of dipole motion were manipulated as a way to limit (Experiment 1) or favour (Experiment 2) the availability of orientation signals from motion (motion streaks). The results of Experiment 1 showed that apparent GP motion direction is attracted towards dipole orientation, and apparent GP orientation is repulsed from GP motion. The results of Experiment 2 showed stronger repulsion effects when judging the GP orientation, but stronger motion streaks from the GP motion can dominate over the signals provided by conflicting dipole orientation. These results are consistent with the proposal that two separate mechanisms contribute to our perception of stimuli which contain conflicting orientation and motion information: (i) perceived GP motion is mediated by spatial motion-direction sensors, in which signals from motion sensors are combined with excitatory input from orientationtuned sensors tuned to orientations parallel to the axis of GP motion, (ii) perceived GP orientation is mediated by orientation-tuned sensors which mutually inhibit each other. The two mechanisms are revealed by the different effects of conflict angle and dipole lifetime on perceived orientation and motion direction.
\end{abstract}

Keywords: Moving Glass patterns, oriented Glass patterns, motion-form interaction, spatial motion-direction sensor, motion streaks 


\section{Introduction}

It has been long established that the visual system of mammals is integrated structurally, whereby different forms of visual information are processed by distinct specialised areas in the brain (Calabretta \& Parisi, 2005). Consistent with this view, it has been proposed that orientation and motion information are processed by separate neural pathways originating in the primary visual cortex, known as the ventral stream and dorsal stream; the ventral stream processing mainly form information and the dorsal stream mainly encoding motion information (Benson \& Greenberg, 1969; Ungerleider \& Mishkin, 1982; Zihl et al., 1983, 1991; Van Essen \& Gallant, 1994; Braddick et al., 2000).

Recent evidence, however, is inconsistent with the recognised view of two parallel, integrated processing streams for form and motion. Evidence instead provides support for the notion that there is widespread interaction between orientation and motion pathways and that these are not separate (Krekelberg et al., 2003; Kourtzi et al., 2008; Or et al., 2010; Pavan et al., 2011, 2013, Mather et al., 2012, 2013). For instance, Lennie (1998) found that while many MT cells in macaque monkey cortex respond strongly to motion information, they also respond to a smaller degree to orientation, implying cells aggregate visual information and interact early on. Other evidence comes from research using Glass patterns (GPs; Glass, 1969), comprising an ensemble of randomly positioned dot-pairs (i.e., dipoles). The pooling of local orientation information, given from the way the dipoles are aligned within the GP, affords the perception of global form (Wilson \& Wilkinson, 1998). Using dynamic GPs (a series of static GPs shown in rapid succession, differing randomly in position), apparent motion can also be perceived along an axis parallel to dipole orientation (Ross et al., 2000), which cannot be differentiated from real motion (Krekelberg et al., 2005). By conveying different angles for motion direction and orientation, GPs can be constructed to produce a conflict between motion and orientation signals (i.e., a conflict angle; the dipoles are given a spatial displacement over time along an axis that is not parallel to dipole orientation). Krekelberg et al. (2003) found that in both humans and macaque monkeys the perceived motion direction of GPs was significantly attracted towards dipole orientation at a small conflict angle (between $10^{\circ}$ and $45^{\circ}$ ). This indicates that perceived motion is established from the weighted average of the real motion direction of the GP and global orientation of the texture. 
Later studies reported that judgements of GP motion direction become less accurate when there is a small conflict angle with dipole orientation, accompanied by an attraction. In addition, findings also demonstrate that perceived global orientation is influenced by motion direction, but to a lesser degree (Or et al., 2010). These results suggest that motion and orientation information interact asymmetrically, whereby the influence of global orientation information on perceived global motion direction is stronger than the reverse. On the basis of such research it cannot be disputed that motion and orientation information are processed by completely independent systems. Instead they interact at early stages within the visual system (Wilson \& Wilkinson, 1998; Dakin \& Bex, 2001; Or et al., 2010).

Over time, summation of responses to a moving visual object, when it is moving with adequate speed, produces "speed" lines that extend backwards across the retina from the object and display the character of the movement (Burr, 1980; Burr \& Ross, 2002), due to temporal integration (Geisler, 1999). The aforementioned findings support the proposition made by Geisler (1999): the dipoles within dynamic GPs simulate "motion streaks" offering cues as to the axis of the motion and influencing the observer's perception of direction, resulting in deflection and attraction effects (Ross et al., 2000; Burr \& Ross, 2002; Ross, 2004). In this way, the processing orientation and motion information supplement one another to make precise direction judgements, particularly for stimuli with higher temporal frequency (Geisler, 1999).

Although previous studies of perceived GP motion and orientation, as a function of conflict angle, indicate an asymmetrical interaction (Krekelberg et al., 2003; Or et al., 2010), the neural mechanisms of the two effects (i.e., how motion affects orientation judgments and vice versa) are still unclear. Using oriented and moving GPs, and varying the angle between dipoles' motion direction and their orientation we assessed the underlying mechanisms of motion-form and how these mechanisms interact. The duration of the stimuli was also manipulated to assess whether the integration between motion and form depends on exposure time. 


\section{Experiment 1}

\section{Methods}

Participants

Two of the authors (AP and MGG) and seven naïve participants took part in the experiment with no compensation. All participants had normal or corrected to normal visual acuity. Viewing was binocular. Methods conformed with the Declaration of Helsinki (1964). The experiment was approved by the ethical committee of the University of Lincoln (reference number: PSY1415124). All participants gave written informed consent prior to their inclusion in the experiment.

\section{Apparatus}

Stimuli were displayed on a 20-inch HP p1230 monitor with a refresh rate of $85 \mathrm{~Hz}$. Stimuli were generated with Matlab PsychToolbox (Brainard, 1997; Pelli, 1997). The screen resolution was $1280 \times 1024$ pixels. Each pixel subtended 1.6 arcmin. The minimum and maximum luminances of the screen were 0.08 and $74.6 \mathrm{~cd} / \mathrm{m}^{2}$ respectively, and the mean luminance was $37.5 \mathrm{~cd} / \mathrm{m}^{2}$. A gamma-corrected lookup table was used so that luminance was a linear function of the digital representation of the image. Observers sat in a dark room at a distance of $57 \mathrm{~cm}$ from the screen. The participant's head was stabilized by asking her/him to rest her/his chin on a chinrest.

\section{Stimuli and Procedure}

Stimuli were oriented translational GPs made up by 134 pairs of white dots (dipoles) with an inter-dot separation of $0.27 \mathrm{deg}$. The contrast of each dot was 0.99 (Weber contrast). The diameter of each dot was $0.07 \mathrm{deg}$. The dipoles were randomly placed in a circular window with a radius of $5.7 \mathrm{deg}$. The dipole density of the GPs was $1.31 \mathrm{dipoles} / \mathrm{deg}^{2}$ (Or et al., 2010). All dipoles had the same orientation (i.e., 100\% coherence). In each trial dipoles moved either leftward or rightward. The motion sequence was computed as follows: on the first frame dipoles were randomly positioned within the circular window and were displaced by $0.063 \mathrm{deg}$ on each subsequent frame, producing a speed of $5.33 \mathrm{deg} / \mathrm{s}$ (similar to Or et al., 2010). Dipoles had a limited lifetime; that is, after 3 frames (i.e., $\sim 0.035 \mathrm{~s}$ ) each dipole vanished and was replaced by a new dipole, with the same orientation, at a different 
randomly selected position within the circular window. Dipoles appeared asynchronously on the display. Limited lifetime and asynchronous presentation were implemented to minimize the presence of local "motion streaks" (Geisler, 1999) that could interfere with orientation judgements. In addition, moving dipoles that travelled outside the circular window were also replaced by a new dipole at a different randomly location within the circular window, thus always maintaining the same dipole density. On a trial-by-trial basis the GP orientation was varied. For rightward motion GP orientations were: $0^{\circ}$ (i.e., motion direction and GP orientation were parallel), $20^{\circ}, 40^{\circ}, 80^{\circ}, 340^{\circ}, 320^{\circ}, 280^{\circ}$ (Figure 1; dashed line was not actually presented). For leftward motion GP orientation were: $180^{\circ}$ (parallel), $160^{\circ}, 140^{\circ}$, $100^{\circ}, 200^{\circ}, 220^{\circ}$ and $260^{\circ}$. Stimulus duration was also varied on a trial-by-trial basis and could be of 6, 12, 24, 48, 96 and 192 frames (corresponding to 0.070, 0.141, 0.282, 0.565, 1.129 , and $2.259 \mathrm{~s})$.
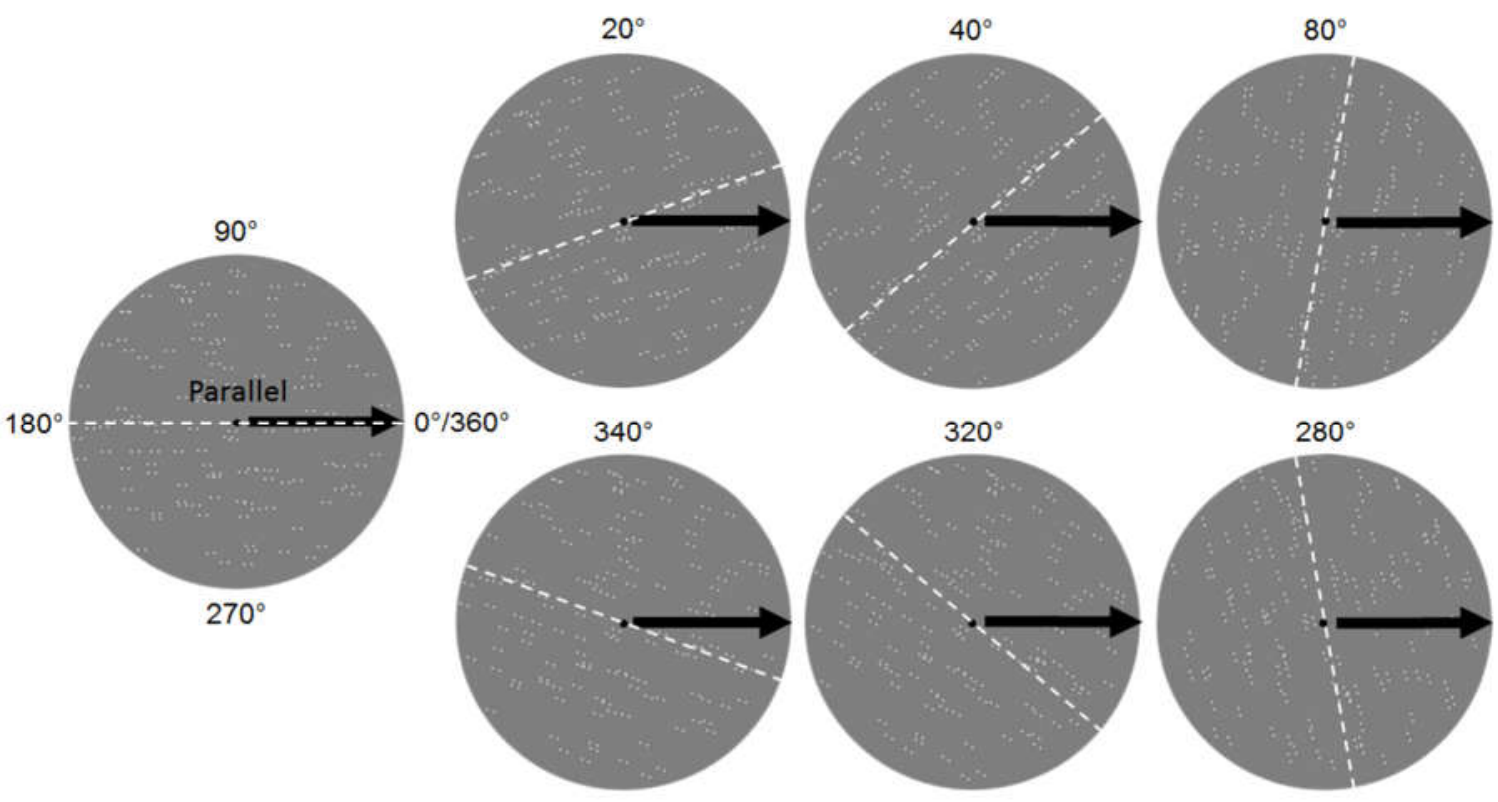

Figure 1. Representation of the stimuli used in Experiment 1. Only rightward motion is showed (thick black arrow) and the relative orientation of the Glass patterns. The white dashed line indicates the orientation of the Glass patterns, and was not shown during the experiment. 
Following presentation of a fixation point for $1 \mathrm{~s}$, the moving GP was presented.

There were 16 orientations x 6 stimulus durations. Each condition was repeated 6 times. The horizontal orientations (i.e., $0 / 360^{\circ}$ and $180^{\circ}$ ) were repeated twice, to have the same number or repetitions as the other orientations. Each observer performed a total of 576 trials split over 12 blocks. The observers performed either orientation judgments or motion direction judgments in a given block (576 trials each). The type of task (i.e., either orientation or motion judgements) was randomized across observers; that is, participants completed first the orientation blocks and then the motion blocks, or vice versa. Observers judged the perceived orientation or motion direction of the GP by setting the position of a line (Figure 2) around a circumference. The initial position of the line was randomized on a trial basis and could be located at any angle between $0^{\circ}$ and $359^{\circ}$.

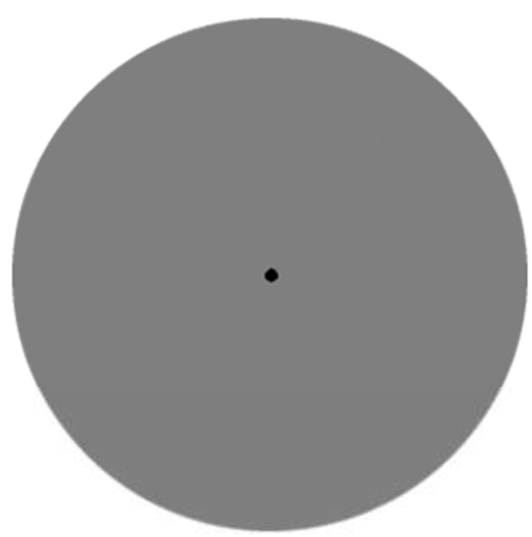

Fixation point (1 s)
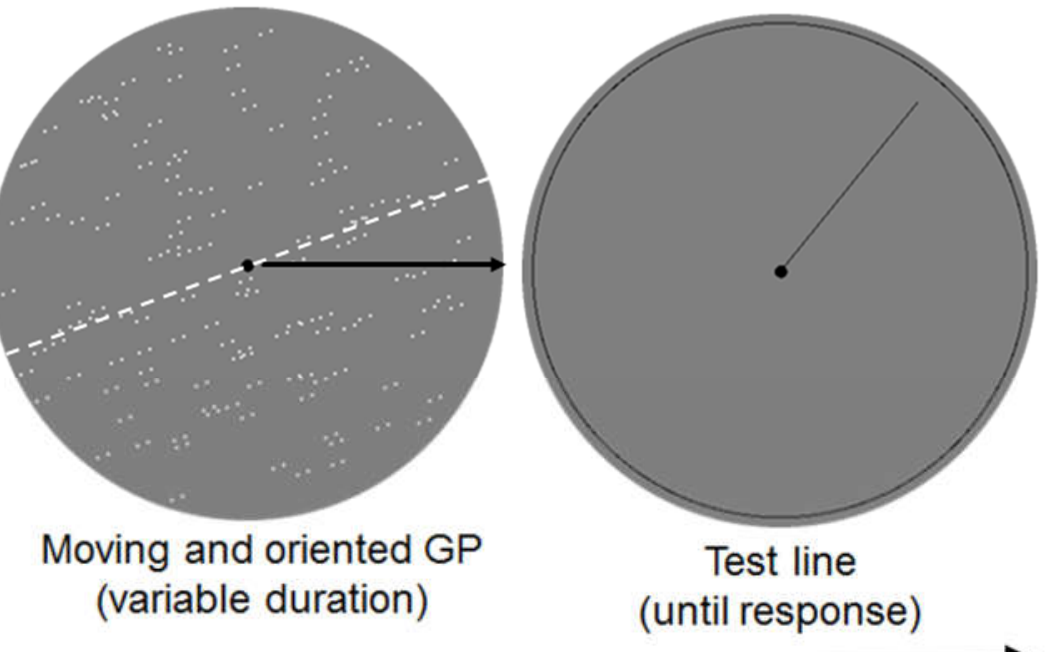

Time

Figure 2. Procedure used in Experiment 1. After initial fixation for $1 \mathrm{~s}$, a rightward moving Glass pattern oriented $20^{\circ}$ from horizontal is presented (variable duration; the white dashed line and the black arrow were not present during the experiment). Observers had to set the line shown in the rightmost panel according to the perceived Glass pattern's orientation or motion direction. 
Data from symmetrical orientation were pooled to get four conflict angles: $0^{\circ}, 20^{\circ}$, $40^{\circ}$, and $80^{\circ}$. The $0^{\circ}$ conflict angle was created by pooling data from the parallel conditions (i.e., $0^{\circ}$ and $180^{\circ}$ ), the $20^{\circ}$ conflict angle was created by pooling data from $20^{\circ}, 340^{\circ}, 200^{\circ}$ and $160^{\circ}$, the $40^{\circ}$ conflict angle was created by pooling data from $40^{\circ}, 320^{\circ}, 220^{\circ}, 140^{\circ}$ and the $80^{\circ}$ conflict angle was created by pooling data from $80^{\circ}, 260^{\circ}, 280^{\circ}$ and $100^{\circ} \mathrm{GP}$ orientation.

\section{Results}

Orientation

Figure 3 (A, B) reports the deflection (i.e., the difference in angular orientation) between the perceived orientation and the actual GP orientation as a function of the conflict angle. Positive values indicate repulsion from the axis of motion, and negative values indicate attraction. A repeated measures ANOVA including as factors the stimulus duration and the conflict angle reported only a significant effect of the conflict angle $\left(F_{1.52,12.17}=16.15, p=\right.$ 0.001 , partial $\left.-\eta^{2}=0.67\right)$. Simple within-subjects contrasts reported that the deflection estimated for conflict angles of $20^{\circ}, 40^{\circ}$ and $80^{\circ}$ was significantly different from the deflection estimated for the $0^{\circ}$ conflict angle (i.e., parallel condition) $\left(20^{\circ}\right.$ vs. $0^{\circ}: F_{1,8}=$ $19.19, p=0.002$, partial $-\eta^{2}=0.71 ; 40^{\circ}$ vs. $0^{\circ}: F_{1,8}=17.84, p=0.003$, partial $-\eta^{2}=0.69 ; 80^{\circ}$ vs. $0^{\circ}: F_{1,8}=10.55, p=0.012$, partial- $\left.\eta^{2}=0.57\right)$. Repeated contrasts did not report a significant difference between deflections at $20^{\circ}$ and $40^{\circ}\left(F_{1,8}=0.155, p=0.70\right.$, partial $-\eta^{2}=$ 0.019). In particular, for $20^{\circ}$ and $40^{\circ}$ conflict angles we found repulsion effects (values above zero); that is, the perceived orientation of the GP was repelled away from the axis of motion (conflict angle $20^{\circ}:$ Mean $=4.9^{\circ}, \mathrm{SEM}=1.26^{\circ}$; conflict angle $40^{\circ}:$ Mean $=4.08^{\circ}, \mathrm{SEM}=$ $0.99^{\circ}$ ), whereas for $80^{\circ}$ conflict angle we found an attraction effect (values below zero), i.e., the perceived orientation of the GP was attracted towards the horizontal motion direction $\left(\right.$ Mean $\left.=-6.41^{\circ}, \mathrm{SEM}=1.87^{\circ}\right)$.

An additional analysis of variance for repeated measures with trend analysis on data pooled across the stimulus durations, revealed significant linear $\left(F_{1,8}=8.28, p=0.021\right.$, partial- $\left.\eta^{2}=0.51\right)$ and quadratic $\left(F_{1,8}=22.85, p=0.001\right.$, partial $\left.-\eta^{2}=0.74\right)$ trends. Higher order trends were not significant. In order to estimate the peak deflection of the perceived orientation, we fitted a quadratic polynomial on mean data $\left(R^{2}=0.98\right)$. The peak (vertex) was 
estimated at a conflict angle of $32.4^{\circ}$ with a deflection between perceived orientation and GP orientation of $4.93^{\circ}$.

\section{Motion}

Figure $3(\mathrm{C}, \mathrm{D})$ reports the deflection between the perceived motion direction and the actual motion direction of the GP as a function of the conflict angle. A repeated measures ANOVA including as factors the stimulus duration and the conflict angle reported only a significant effect of the conflict angle $\left(F_{3,24}=6.67, p=0.002\right.$, partial- $\left.\eta^{2}=0.46\right)$. Simple within-subjects contrast reported that deflections estimated for conflict angles of $40^{\circ}$ and $80^{\circ}$ were significantly different from the deflection estimated for the $0^{\circ}$ conflict angle $\left(40^{\circ} \mathrm{vs} .0^{\circ}\right.$ : $F_{1,8}=13.13, p=0.007$, partial $-\eta^{2}=0.62 ; 80^{\circ}$ vs. $0^{\circ}: F_{1,8}=23.39, p=0.001$, partial $-\eta^{2}=$ 0.75). Repeated contrasts pointed out a significant difference between conflict angles of $20^{\circ}$ and $40^{\circ}\left(F_{1,8}=5.35, p=0.05\right.$, partial $\left.-\eta^{2}=0.4\right)$. An analysis of variance for repeated measures with trend analysis revealed a significant linear trend only for the conflict angle $\left(F_{1,8}=22.97, p=0.001\right.$, partial $\left.-\eta^{2}=0.74\right)$. A linear regression on mean data (Figure 3D) confirmed a linear relationship between estimated deflections on motion judgements and conflict angle $\left(R^{2}=0.92\right)$. 
A

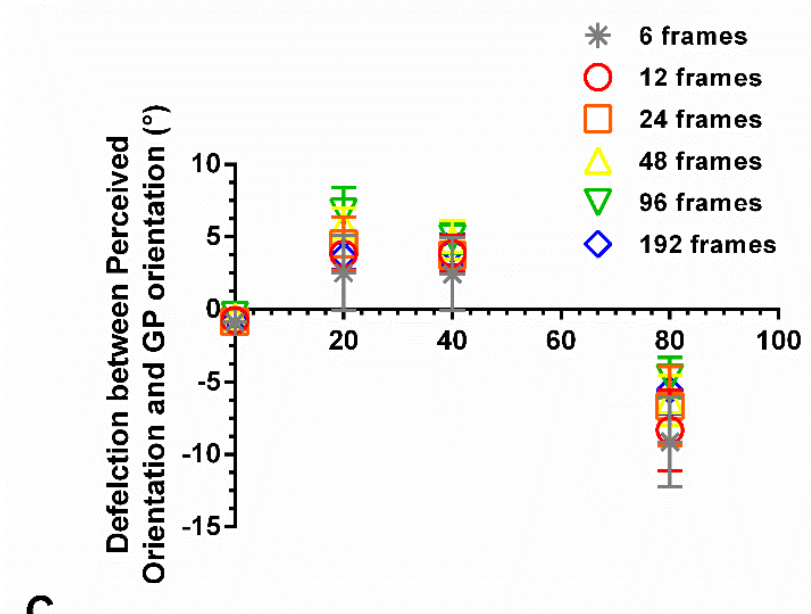

C

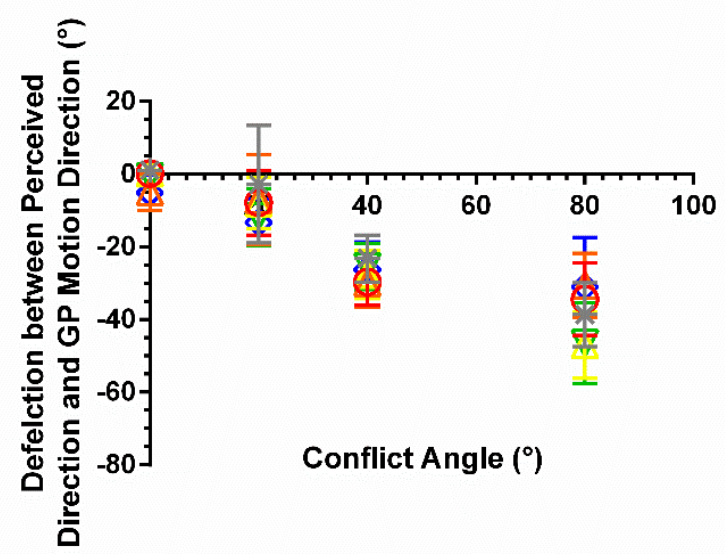

B
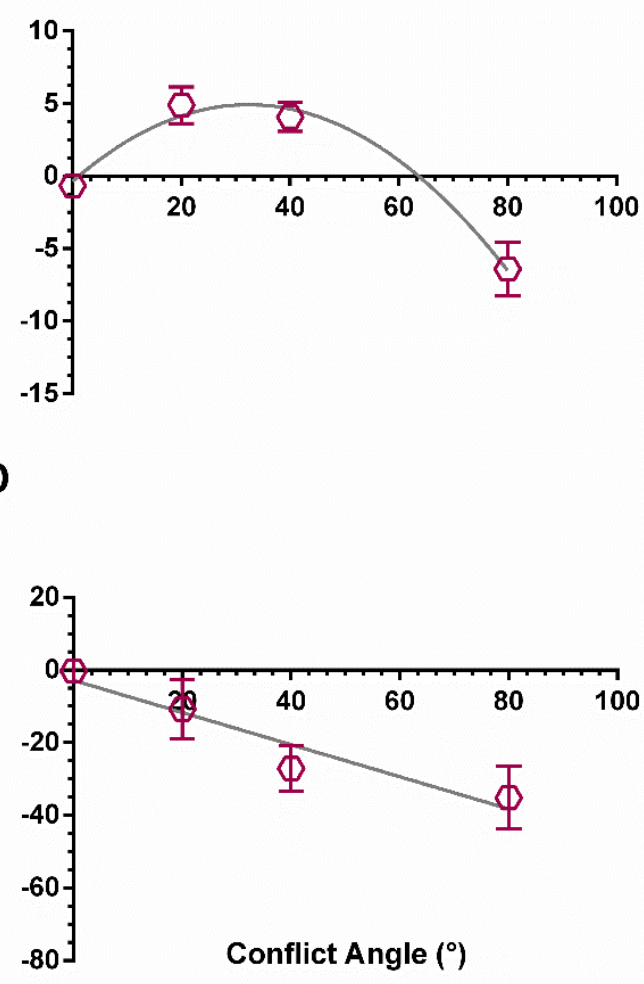

Figure 3. Results of Experiment 1. Panels A and B: orientation judgments. Deflection, i.e., difference $\left(^{\circ}\right)$ between the perceived orientation and the actual GP orientation is shown as a function of the conflict angles used. (A) Deflection values are reported separately for each stimulus duration (in frames). (B) Mean deflection across the stimulus durations used. The grey curve indicates a quadratic polynomial fitted on the mean data $\left(f(x)=a x^{2}+b x+c ; a\right.$ $=-0.005[95 \% \mathrm{CI}=-0.014,0.004], b=0.327[95 \% \mathrm{CI}=-0.43,1.08], c=-0.367[95 \% \mathrm{CI}=-$ 12.3, 11.56]). Panels C and D: motion judgments. (C) Deflection values are reported separately for each stimulus duration. (D) Mean deflection across the stimulus durations used. The continuous grey line indicates a linear regression on mean data $(f(x)=a x+b ; a=$ $-0.44[95 \% \mathrm{CI}=-0.839,-0.045], b=-2.87[95 \% \mathrm{CI}=-21.5,15.3])$. Values above zero indicate repulsion whereas values below zero indicate attraction. Error bars \pm SEM. 


\section{Discussion}

The results of Experiment 1 show that apparent GP motion is attracted towards dipole orientation, and the attraction increases monotonically with conflict angle; apparent GP orientation is repulsed from GP motion, with repulsion peaking at intermediate conflict angles. The effect of orientation on motion was much more marked than the effect of motion on orientation (note the y-axis scales in Figure 3), consistent with the data of Or et al. (2010).

One could argue that such a strong asymmetry between orientation and motion, with orientation dominating over motion direction, could be due to the weaker global motion signals originating when using a very short lifetime (i.e., $0.035 \mathrm{~s}$ ). In a control experiment, we tested for this possibility by measuring the strength of the motion signals from globally moving random dot kinematograms (RDKs), in which dots had the same spatiotemporal characteristics of the moving dipoles used in Experiment 1.

\section{Control Experiment}

\section{Methods}

\section{Participants}

Two of the authors (AP and FG) and four naïve participants took part voluntarily to the experiment with no compensation. All participants had normal or corrected to normal visual acuity. Viewing was binocular. All participants gave written informed consent prior to their inclusion in the experiment.

\section{Stimuli and Procedure}

Stimuli and procedure were the same as in Experiment 1, but instead of moving and oriented GPs, observers had to judge the motion direction of RDKs composed by globally moving dots with the same spatiotemporal characteristics of the moving dipoles reported in Experiment 1. It should be noted that RDKs had the same density of GPs, but dipoles were no longer present. Observers had to set the line (Figure 2, rightmost panel) accordingly to the perceived motion direction. There were only two motion directions (i.e., leftward and rightward motion), but observers were not informed about the possible motion directions of the RDKs. We considered a correct response when the line was set in the half circle consistent with the RDK's motion direction. 


\section{Results}

Figure 4 shows the results of the control experiment. A repeated measures ANOVA on proportion of correct responses including as factors the motion direction and the stimulus duration, reported only a significant effect of the duration $\left(F_{1.6,8.01}=6.59, p=0.024\right.$, partial$\eta^{2}=0.57$ ). Post-hoc comparisons using a false discovery rate (FDR) at 0.05 (Benjamini \& Hochberg, 1995; Benjamini \& Yekutieli, 2001, 2005) reported that the 6-frame duration was significantly different from the 12, 24, and 48-frame conditions (adjusted- $p=0.005,0.005$, and 0.022 , respectively). In order to assess whether mean proportion of correct responses were significantly above the chance level (0.5), we performed a series of one-sample t-tests using a FDR at 0.05 . The t-tests reported that mean proportion of correct responses were significantly above chance for all the durations used (adjusted- $p$ values: 0.049, 0.015, 0.015, 0.015, 0.049 and 0.049, respectively for $6,12,24,48,96$, and 192-frame conditions).

A repeated measures ANOVA conducted on the deflection angles (Figure 4B, i.e., angular difference between the perceived motion direction and the actual RDKs' motion direction) for correct responses, did not report any significant effect or interaction (motion direction: $F_{1,5}=0.34, p=0.59$, partial $-\eta^{2}=0.063$; duration: $F_{2.18,10.89}=0.34, p=0.74$, partial $-\eta^{2}=0.064$; interaction motion direction $\mathrm{x}$ duration: $F_{2.20,11}=1.60, p=0.25$, partial- $\eta^{2}$ $=0.24)$. In addition, one-sample t-tests using a FDR at 0.05 reported that no deflections values were significantly different from zero (adjusted- $p>0.05$ ).

A

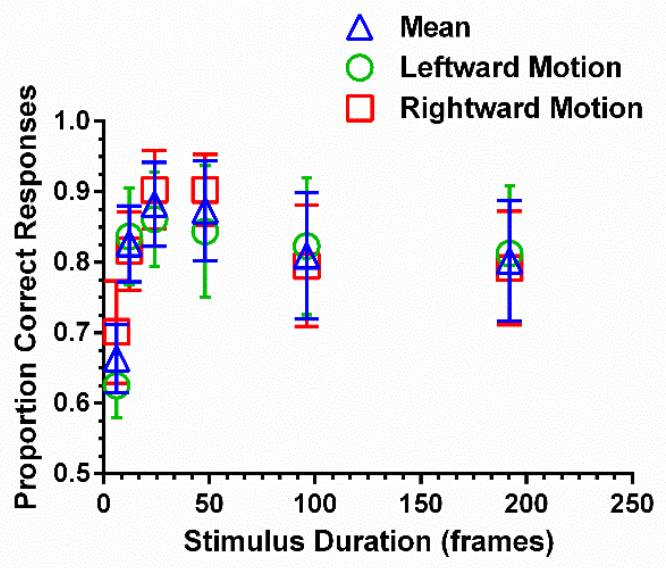

B

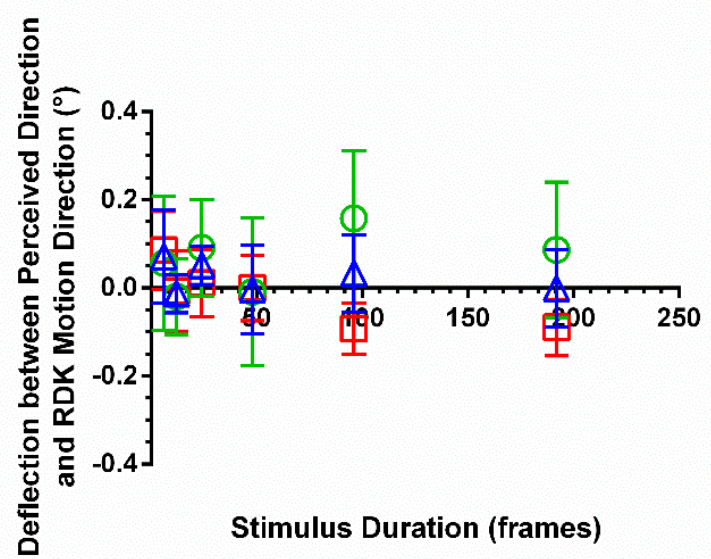


Figure 4. Results of the control experiment. (A) Proportion of correct judgements for leftward and rightward motion. (B) Mean deflection angles $\left(^{\circ}\right)$ between the perceived motion direction and $180^{\circ}$ for leftward motion, and $0^{\circ} / 360^{\circ}$ for rightward motion. Deflection values are reported only for correct responses. Error bars \pm SEM.

\section{Discussion}

The results of the control experiment suggest that global motion signals were reliable across all the durations employed. Observers could discriminate above chance the motion direction of the globally moving RDKs, though for the shortest duration the mean proportion of correct responses was 0.66 (SEM: 0.05), but still significantly different from chance. Therefore, the orientation dominance reported in Experiment 1 was unlikely to be due to weak and negligible global motion signals.

However, in Experiment 2 we increased the dipole lifetime (from 0.035 to $0.494 \mathrm{~s}$ ) in order to induce stronger "motion streaks" from moving dipoles (Geisler, 1999; Apthorp \& Alais, 2009). The rationale was to assess the interaction between orientation and motion with dipoles inducing motion streaks along their axis of motion, thus further aiding the extraction of motion. If the induction of motion streaks was successful, one would expect a greater influence of motion on orientation judgements, and only a weak or no effect of orientation on motion judgments.

\section{Experiment 2}

\section{Methods}

Four of the authors (AP, LMB, MGG and FG) and seven naïve observers participated to Experiment 2. Apparatus, stimuli and procedure were the same as Experiment 1, except that dipole lifetime was 42 frames (i.e., $0.494 \mathrm{~s}$ ). Moreover, we used five of the stimulus durations used in Experiment 1 (i.e., 12, 24, 48, 96 and 192 frames, corresponding to 0.141, $0.282,0.565,1.129$, and $2.259 \mathrm{~s})$. All the other spatiotemporal characteristics of the stimulus were the same to that of Experiment 1. Based on Geisler (1999) if the speed of a dot exceeds about 1 dot width per $0.1 \mathrm{~s}$, it produces a greater response in the population of neurons whose receptive fields are oriented parallel to the direction of motion. In our case, the speed of the dots composing a dipole exceeded the width of a $\operatorname{dot}(0.07 \mathrm{deg})$ in $0.1 \mathrm{~s}(\sim 0.53 \mathrm{deg}$ in $0.1 \mathrm{~s})$, 
therefore the speed and the dipoles' lifetime employed were likely to generate motion smear.

\section{Results}

\section{Orientation}

Figure 5 (A, B) reports the deflection angles between the perceived orientation and the actual GP orientation as a function of the conflict angles used. A repeated measures ANOVA including as factors the stimulus duration and the conflict angle reported only a significant effect of the conflict angle $\left(F_{3,30}=10.62, p=0.0001\right.$, partial $\left.-\eta^{2}=0.52\right)$. Simple within-subjects contrasts reported that the deflection estimated for conflict angles of $20^{\circ}$ and $40^{\circ}$ was significantly different from the deflection estimated for the $0^{\circ}$ conflict angle $\left(20^{\circ} \mathrm{vs}\right.$. $0^{\circ}: F_{1,10}=10.13, p=0.010$, partial $-\eta^{2}=0.50 ; 40^{\circ}$ vs. $0^{\circ}: F_{1,10}=13.48, p=0.004$, partial $-\eta^{2}$ $=0.57$ ). However, deflections estimated for $80^{\circ}$ and $0^{\circ}$ conflict angles were not significantly different $\left(F_{1,10}=0.95, p=0.35\right.$, partial $\left.-\eta^{2}=0.087\right)$. Repeated contrasts did not report a significant difference between deflections estimated at $20^{\circ}$ and $40^{\circ}$ conflict angles $\left(F_{1,8}=\right.$ $0.155, p=0.70$, partial $-\eta^{2}=0.019$ ). In particular, we found repulsion effects for $20^{\circ}$ and $40^{\circ}$ conflict angles (values above zero); that is, the perceived orientation of the GP was repelled away from its actual orientation (conflict angle $20^{\circ}: \mathrm{M}=5.56^{\circ}$, SEM: $1.55^{\circ}$; conflict angle $40^{\circ}: \mathrm{M}=8.05^{\circ}$, SEM: $1.77^{\circ}$ ), whereas for $80^{\circ}$ conflict angle we did not find any significant repulsive or attractive effects. An additional analysis of variance for repeated measures with trend analysis on data pooled across the stimulus duration, revealed a significant quadratic trend $\left(F_{1,10}=23.57, p=0.001\right.$, partial $\left.-\eta^{2}=0.70\right)$. In order to estimate the peak deflection of the perceived orientation, we fitted a quadratic polynomial $\left(R^{2}=0.99\right)$. The peak (vertex) was estimated at a conflict angle of $38.8^{\circ}$ with a deflection between perceived orientation and GP orientation of $7.8^{\circ}$.

\section{Motion}

Figure $5(\mathrm{C}, \mathrm{D})$ reports the deflection between the perceived motion direction and the actual motion direction of the GP as a function of the conflict angle. A repeated measures ANOVA including as factors the stimulus duration and the conflict angle reported only a significant interaction between duration and conflict angle $\left(F_{12,120}=1.88, p=0.043\right.$, partial$\eta^{2}=0.16$ ). Post-hoc comparisons using a FDR at 0.05 reported only a significant difference 
between durations of 24 and 48 frames at conflict angle of $40^{\circ}$ (adjusted- $p=0.048$ ).

A

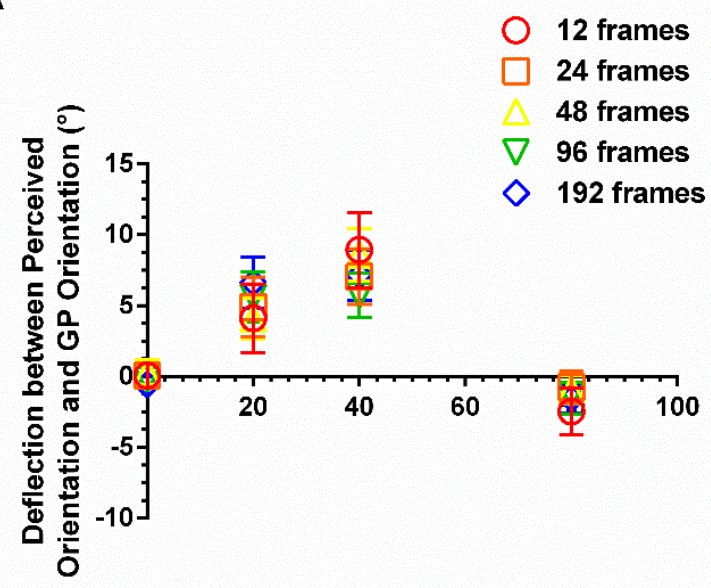

C

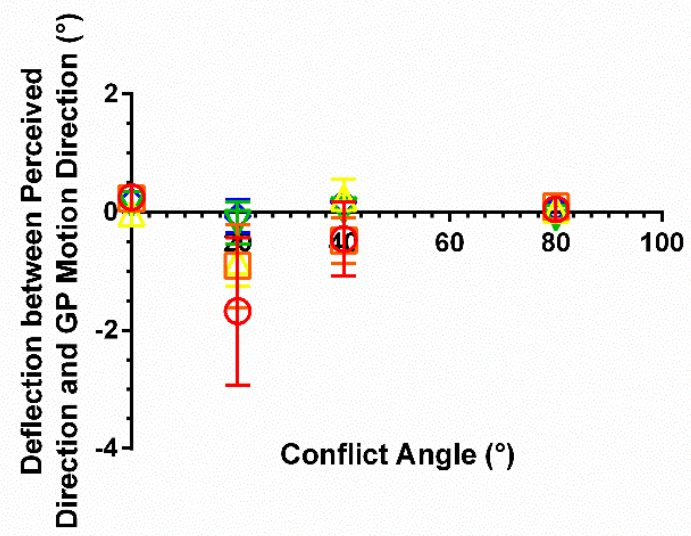

B

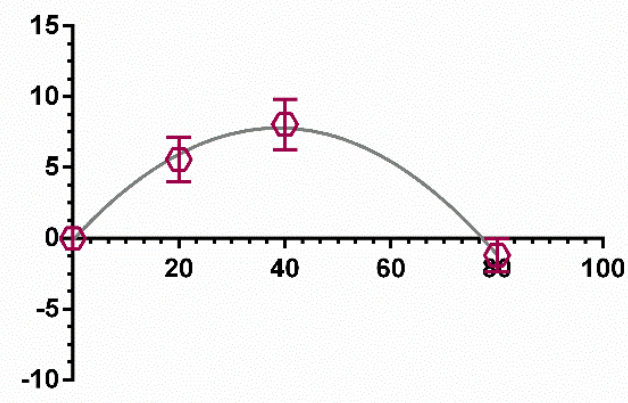

D

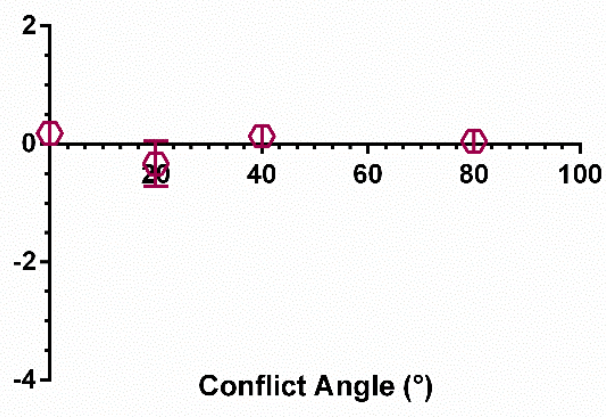

Figure 5. Results of Experiment 2. Panels A and B: orientation judgments. (A) Deflection values are reported separately for each stimulus duration (in frames). (B) Mean deflection across the stimulus durations used. The grey curve indicates a quadratic polynomial fitted on the mean data $(a=-0.0053[95 \% \mathrm{CI}=-0.009,0.001], b=0.408[95 \% \mathrm{CI}=0.043,0.774], c=-$ $0.139[95 \% \mathrm{CI}=-5.904,5.625])$. Values above zero indicate repulsion whereas values below zero indicate attraction. Panels C and D: motion judgments. (C) Deflection values are reported separately for each stimulus duration. (D) Mean deflection across the stimulus durations used. Values above zero indicate repulsion whereas values below zero indicate attraction Error bars \pm SEM. 


\section{Discussion}

The results of Experiment 2 show that apparent repulsion of orientation was greater in conditions which produced stronger orientation signals from streaks. In addition, the stronger streaks from the GP motion allowed the orientation signals they generated to dominate over the signals provided by conflicting dipole orientation.

\section{General Discussion}

In two experiments, we investigated the integration between motion and form and its temporal dynamics. Considered together, our results are consistent with the presence of two separate mechanisms that contribute to our perception of Glass patterns which contain conflicting orientation and motion information.

The effect of dipole orientation on apparent GP motion direction is likely to be mediated by Geisler's (1999) "spatial motion-direction sensor" (SMD sensor), in which signals from motion sensors are combined with excitatory input from orientation-tuned sensors, which are tuned to orientations that are parallel to the axis of GP motion. This model predicts that the perceived GP motion direction should be attracted towards dipole orientation, and that the bias in apparent direction should increase with the conflict angle due to the increasing bias introduced at larger conflict angles (Krekelberg et al., 2003). The results of Experiment 1 are consistent with this model showing that the bias in apparent GP direction monotonically increases as the conflict angle increases. Besides, the same model predicts that the attraction should be weaker (or absent) in conditions which are optimal for producing streaks generated by the motion itself (the parallel streak signals become dominant over the non-parallel signals from the dipole orientation). The results of Experiment 2 for motion judgements are also consistent with this prediction. Findings suggest that SMD sensors may therefore be present at both low- and intermediate levels of visual processing. Albright (1984) investigated the orientation selectivity of 89 MT cells of macaque monkey and found that $29 \%$ of the cells exhibited an orientation preference that is roughly parallel to the preferred motion direction. More recently, a human brain imaging study by Apthorp et al. (2013) showed that motion streaks are likely to be extracted at early stages of visual analysis, implying that motion and form, while seemingly separate, are processed and combined as early as the primary visual cortex. In addition, there is brain imaging and psychophysical 
evidence that early visual areas can encode and pool the sparse local orientation cues in translational GPs (Ohla et al., 2005; Ostwald et al., 2008; Mannion et al., 2009, 2010; Pavan et al., 2016).

On the other hand, the effect of GP motion on apparent dipole orientation is likely to be mediated by interactions between orientation-tuned cells responding to the orientations of the dipole and the streak generated by dipole motion (Geisler, 1999; Apthorp \& Alais, 2009). This mechanism predicts that apparent dipole orientation should be repulsed away from the axis of GP motion due to mutual inhibition between orientation-tuned sensors, but the repulsion should peak at intermediate conflict angles, as expected on the basis of data from studies of orientation interactions and adaptation (Apthorp et al., 2011). The results of Experiment 1 for orientation judgments showed a repulsion effect peaking at a conflict angle of $32.4^{\circ}$, with a bias of $4.93^{\circ}$ (see Figures $3 \mathrm{~A}$ and $3 \mathrm{~B}$ ). Furthermore, this model predicts that the repulsion should be even stronger in conditions which are optimal for producing streaks (orientation signals) generated by the motion itself. The results of Experiment 2 are also consistent with this model and showed a larger bias of approximately $8^{\circ}$ at a conflict angle of $39^{\circ}$ (see Figures 5A and 5B). Or et al. (2010) found a similar repulsion effect with conflict angles higher than $30^{\circ}$ when observers were required to judge the perceived orientation of moving GPs. The repulsion effects reported are similar to those present in motion and orientation domain (Marshak \& Sekuler, 1979; Mather \& Moulden, 1980; Burke \& Wenderoth, 1993; Qian \& Geesaman, 1995; Hiris \& Blake, 1996; Kim \& Wilson, 1996; Rauber \& Treue, 1998; Wishart et al., 1998; Benton \& Curran, 2003; Chen et al., 2005). When two random fields of dots drift in two different directions, or two intersecting lines are presented, the perceived directions and orientations repel each other for conflict angles between 20 and $40^{\circ}$, but attract each other at larger conflict angles (Marshak \& Sekuler, 1979; Rauber \& Treue, 1998). Accordingly, in the first experiment, we found a repulsion effect of $\sim 5^{\circ}$ at a conflict angle of approximately $32^{\circ}$, however, for the highest conflict angle used $\left(80^{\circ}\right)$ we found an attraction effect of $6.41^{\circ}$ (Figures 3A and 3B). Such form and formby-motion interaction is likely to occur at a low level of visual analysis, where neurons are more selective for the orientation, and with repulsion between orientations occurring only for small acute angles (Marshak \& Sekuler, 1979).

Importantly, in all the experiments we did not find any effect of the stimulus duration; 
this could depend on feedforward and feedback connections between low- and high-level visual areas encoding and combining orientation and motion signals. For instance, a number of physiological studies on monkeys and brain stimulation studies in humans, suggest the presence of feedback connections from high level visual areas (e.g., MT) to low level areas (e.g., V1/V2). These feedback signals may influence the transfer of ascending input when or before the input arrives to the visual cortex, shaping the perception of moving contours and textures (Sillito et al., 2006; Laycock et al., 2007). Moreover, feedback connections have an important role in the formation of the inhibitory center-surround interactions (for moving surrounds) present at low level of visual analysis and mediate the integration of visual information (Lamme et al., 1998; Bullier et al., 2001).

In summary, our findings suggest that motion and form information are likely to be encoded in parallel and then combined. Moreover, they are subserved by two discrete mechanisms (SMD sensor and orientation-tuned cells) revealed by the different effects of conflict angle and dipole lifetime on perceived orientation and motion direction.

\section{Acknowledgments}

AP, MGG and GM designed the experiments. AP implemented the experiments. MGG and LMB collected the data. AP analysed data. AP and GM interpreted the results. AP, LMB and GM wrote the main manuscript. All authors reviewed the manuscript. The study was supported by the University of Lincoln 


\section{References}

Albright TD (1984). Direction and orientation selectivity of neurons in visual area MT of the macaque. J Neurophysiol 52, 1106.

Apthorp D \& Alais D (2009). Tilt aftereffects and tilt illusions induced by fast translational motion: Evidence for motion streaks. $J$ Vis 9, 1-11.

Apthorp D, Cass J \& Alais D (2011). The spatial tuning of "motion streak" mechanisms revealed by masking and adaptation. J Vis 11, 17.

Apthorp D, Schwarzkopf DS, Kaul C, Bahrami B, Alais D \& Rees G (2013). Direct evidence for encoding of motion streaks in human visual cortex. Proc Biol Sci 280, 20122339.

Benson D \& JP G (1969). Visual form agnosia: A specific defect in visual discrimination. Arch Neurol 20, 82-89.

Benton CP \& Curran W (2003). Direction repulsion goes global. Curr Biol 13, 767-771.

Braddick OJ, O’Brien JMD, Wattam-Bell J, Atkinson J \& Turner R (2000). Form and motion coherence activate independent, but not dorsal/ventral segregated, networks in the human brain. Curr Biol 10, 731-734.

Brainard DH (1997). The Psychophysics Toolbox. Spat Vis 10, 433-436.

Bullier J, Hupe JM, James AC \& Girard P (2001). The role of feedback connections in shaping the responses of visual cortical neurons. Prog Brain Res 134, 193-204.

Burke D \& Wenderoth P (1993). Determinants of two-dimensional motion aftereffects induced by simultaneously- and alternately-presented plaid components. Vision Res 33, $351-359$.

Burr D (1980). Motion smear. Nature 284, 164-165. Available at: http://www.ncbi.nlm.nih.gov/pubmed/7360241.

Burr DC \& Ross J (2002). Direct evidence that "speedlines" influence motion mechanisms. $J$ Neurosci 22, 8661-8664.

Calabretta R \& Parisi D (2005). Evolutionary Connectionism and Mind/Brain Modularity. In Modularity: Understanding the Development and Evolution of Natural Complex Systems, pp. 309-330.

Chen Y, Meng X, Matthews N \& Qian N (2005). Effects of attention on motion repulsion. Vision Res 45, 1329-1339.

Dakin SC \& Bex PJ (2001). Local and global visual grouping: Tuning for spatial frequency 
and contrast. $J$ Vis $\mathbf{1}, 99-111$.

Van Essen DC \& Gallant JL (1994). Neural mechanisms of form and motion processing in the primate visual system. Neuron 13, 1-10.

Geisler WS (1999). Motion streaks provide a spatial code for motion direction. Nature 400, $65-69$.

Glass L (1969). Moiré Effect from Random Dots. Nature 223, 578-580.

Hiris E \& Blake R (1996). Direction repulsion in motion transparency. Vis Neurosci 13, 187 197.

Kim J \& Wilson H. (1996). Direction repulsion between components in motion transparency. Vision Res 36, 1177-1187.

Kourtzi Z, Krekelberg B \& van Wezel RJA (2008). Linking form and motion in the primate brain. Trends Cogn Sci 12, 230-236.

Krekelberg B, Dannenberg S, Hoffmann K-P, Bremmer F \& Ross J (2003). Neural correlates of implied motion. Nature 424, 674-677.

Krekelberg B, Vatakis A \& Kourtzi Z (2005). Implied motion from form in the human visual cortex. J Neurophysiol 94, 4373-4386.

Lamme VAF, Supèr H \& Spekreijse H (1998). Feedforward, horizontal, and feedback processing in the visual cortex. Curr Opin Neurobiol 8, 529-535.

Laycock R, Crewther DP, Fitzgerald PB \& Crewther SG (2007). Evidence for Fast Signals and Later Processing in Human V1/V2 and V5/MT+: A TMS Study of Motion Perception. J Neurophysiol 98, 1253-1262.

Lennie P (1998). Single units and visual cortical organization. Perception 27, 889-935.

Mannion DJ, McDonald JS \& Clifford CWG (2009). Discrimination of the local orientation structure of spiral Glass patterns early in human visual cortex. Neuroimage 46, 511-515.

Mannion DJ, McDonald JS \& Clifford CWG (2010). The influence of global form on local orientation anisotropies in human visual cortex. Neuroimage 52, 600-605.

Marshak W \& Sekuler R (1979). Mutual repulsion between moving visual targets. Science (80- ) 205, 1399-1401.

Mather G \& Moulden B (1980). A simultaneous shift in apparent direction: further evidence for a "distribution-shift" model of direction coding. Q J Exp Psychol 32, 325-333.

Mather G, Pavan A, Bellacosa RM \& Casco C (2012). Psychophysical evidence for 
interactions between visual motion and form processing at the level of motion integrating receptive fields. Neuropsychologia 50, 153-159.

Mather G, Pavan A, Bellacosa Marotti R, Campana G \& Casco C (2013). Interactions between motion and form processing in the human visual system. Front Comput Neurosci 7, 65.

Ohla K, Busch NA, Dahlem MA \& Herrmann CS (2005). Circles are different: The perception of Glass patterns modulates early event-related potentials. Vision Res $\mathbf{4 5}$, $2668-2676$.

Or CCF, Khuu SK \& Hayes A (2010). Moving glass patterns: Asymmetric interaction between motion and form. Perception 39, 447-463.

Ostwald D, Lam JM, Li S \& Kourtzi Z (2008). Neural coding of global form in the human visual cortex. J Neurophysiol 99, 2456-2469.

Pavan A, Casco C, Mather G, Bellacosa RM, Cuturi LF \& Campana G (2011). The effect of spatial orientation on detecting motion trajectories in noise. Vision Res 51, 2077-2084.

Pavan A, Hocketstaller J, Contillo A \& Greenlee MW (2016). Tilt aftereffect following adaptation to translational Glass patterns. Sci Rep 6, 23567.

Pavan A, Marotti RB \& Mather G (2013). Motion-form interactions beyond the motion integration level: evidence for interactions between orientation and optic flow signals. $J$ Vis 13, 16.

Pelli DG (1997). The VideoToolbox software for visual psychophysics: transforming numbers into movies. Spat Vis 10, 437-442.

Qian N \& Geesaman BJ (1995). Motion repulsion depends on the distance between the moving elements. $A R V O$.

Rauber HJ \& Treue S (1998). Reference repulsion when judging the direction of visual motion. Perception 27, 393-402.

Ross J (2004). The perceived direction and speed of global motion in Glass pattern sequences. Vision Res 44, 441-448.

Ross J, Badcock DR \& Hayes A (2000). Coherent global motion in the absence of coherent velocity signals. Curr Biol 10, 679-682.

Sillito AM, Cudeiro J \& Jones HE (2006). Always returning: feedback and sensory processing in visual cortex and thalamus. Trends Neurosci 29, 307-316. 
Ungerleider LG \& Mishkin M (1982). Two cortical visual systems. Anal Vis Behav549-586.

Wilson HR \& Wilkinson F (1998). Detection of global structure in Glass patterns: Implications for form vision. Vision Res 38, 2933-2947.

Wishart K., Braddick O. \& Curran W (1998). Direction repulsion in transparent and segregated motions. Investig Ophthalmol Vis Sci 39, 1076.

Zihl J, Von Cramon D \& Mai N (1983). Selective disturbance of movement vision after bilateral brain damage. Brain 106, 313-340.

Zihl J, Von Cramon D, Mai N \& Schmid CH (1991). Disturbance of movement vision after bilateral posterior brain damage: Further evidence and follow up observations. Brain 114, 2235-2252. 